\title{
Task Engagement Selectively Modulates Neural Correlations in Primary Auditory Cortex
}

\author{
(1) Joshua D. Downer, Mamiko Niwa, and Mitchell L. Sutter \\ Center for Neuroscience and Department of Neurobiology, Physiology and Behavior, University of California, Davis, Davis, California 95618
}

\begin{abstract}
Noise correlations $\left(r_{\text {noise }}\right)$ between neurons can affect a neural population's discrimination capacity, even without changes in mean firing rates of neurons. $r_{\text {noise }}$, the degree to which the response variability of a pair of neurons is correlated, has been shown to change with attention with most reports showing a reduction in $r_{\text {noise }}$. However, the effect of reducing $r_{\text {noise }}$ on sensory discrimination depends on many factors, including the tuning similarity, or tuning correlation $\left(r_{\text {tuning }}\right)$, between the pair. Theoretically, reducing $r_{\text {noise }}$ should enhance sensory discrimination when the pair exhibits similar tuning, but should impair discrimination when tuning is dissimilar. We recorded from pairs of neurons in primary auditory cortex (A1) under two conditions: while rhesus macaque monkeys (Macaca mulatta) actively performed a threshold amplitude modulation (AM) detection task and while they sat passively awake. We report that, for pairs with similar AM tuning, average $r_{\text {noise }}$ in A1 decreases when the animal performs the AM detection task compared with when sitting passively. For pairs with dissimilar tuning, the average $r_{\text {noise }}$ did not significantly change between conditions. This suggests that attention-related modulation can target selective subcircuits to decorrelate noise. These results demonstrate that engagement in an auditory task enhances population coding in primary auditory cortex by selectively reducing deleterious $r_{\text {noise }}$ and leaving beneficial $r_{\text {noise }}$ intact.
\end{abstract}

Key words: noise correlation; auditory cortex; population coding; signal correlation; tuning correlation; attention

\section{Introduction}

Understanding how neural populations encode information and how changes in behavioral states affect these codes constitutes an exciting frontier in neuroscience. Many studies report that interneuronal correlations contribute to population coding fidelity (Zohary et al., 1994; Oram et al., 1998; Averbeck et al., 2006; Cohen and Maunsell, 2009). Tuning correlation ( $\mathrm{r}_{\text {tuning }}$ ) quantifies the degree to which two neurons respond similarly to a stimulus set; noise correlation $\left(r_{\text {noise }}\right)$ quantifies the degree to which two neurons' response variability to a given stimulus is correlated. When $r_{\text {tuning }}$ and $r_{\text {noise }}$ have identical sign, sensory discrimination is impaired; and when they have opposite sign, discrimination is enhanced (Oram et al., 1998; Shadlen and Newsome, 1998; Abbott and Dayan, 1999; Nirenberg and Latham, 2003; Romo et al., 2003; Averbeck et al., 2006). Most studies report that both $r_{\text {tuning }}$ and $r_{\text {noise }}$ between pairs of nearby neurons

Received Sept. 29, 2014; revised March 11, 2015; accepted March 25, 2015.

Author contributions: M.N. and M.L.S. designed research; M.N. performed research; J.D.D. contributed unpublished reagents/analytic tools; J.D.D. analyzed data; J.D.D. and M.L.S. wrote the paper.

This work was supported by National Institutes of Health National Institute on Deafness and Other Communication Disorders Grant DC002514 to M.L.S., National Institutes of Health National Research Service Award fellowship F31DC008935 to M.N., and National Science Foundation GRFP fellowship 1148897 to J.D.D. We thank Kevin $\mathrm{O}^{\prime}$ Connor, Doug Totten, and Jessica Verhein for comments on previous versions of the manuscript; and Brittany Rapone for assistance in figure creation.

The authors declare no competing financial interests.

Correspondence should be addressed to Dr. Mitchell L. Sutter, Center for Neuroscience, University of California, Davis, 1544 Newton Court, Davis, CA 95618. E-mail: mlsutter@ucdavis.edu.

M. Niwa's current address: Department of Otolaryngology, Stanford University School of Medicine, Stanford, CA 94305.

DOI:10.1523/JNEUROSCI.4094-14.2015

Copyright $\odot 2015$ the authors $\quad 0270-6474 / 15 / 357565-10 \$ 15.00 / 0$ are on average positive in sensory cortex and that $\mathrm{r}_{\text {noise }}$ remains positive even when $r_{\text {tuning }}$ is negative (Smith and Kohn, 2008; Cohen and Kohn, 2011). Thus, cortex contains a mixture of $\mathrm{r}_{\text {tuning }} / \mathrm{r}_{\text {noise }}$ relationships: some benefit the population code and some impair it.

Behavioral states, such as attention and wakefulness, affect $r_{\text {noise }}$ (Cohen and Newsome, 2008; Mitchell et al., 2009; Herrero et al., 2013; Issa and Wang, 2013; Ecker et al., 2014). Most reports show that, during increased sensory demand (e.g., when attention is required), pairs of neurons decrease $r_{\text {noise }}$, regardless of $\mathrm{r}_{\text {tuning }}$ (Smith and Kohn, 2008; Cohen and Maunsell, 2009). Decreasing $r_{\text {noise }}$ is expected to enhance sensory discrimination for neuron pairs with positive $r_{\text {tuning }}$ but weaken it for pairs with negative $r_{\text {tuning }}$ (e.g., Gu et al., 2011). Recently, however, Jeanne et al. (2013) found that learning decreases $r_{\text {noise }}$ when $r_{\text {tuning }}$ is positive but increases it when $r_{\text {tuning }}$ is negative. This suggests that selective $r_{\text {noise }}$ modulation provides a mechanism not only for learning, but also for rapid changes in sensory discrimination.

The view that primary auditory cortex (A1) is a purely sensory field is changing as recent findings show that myriad behavioral variables affect its activity (Scheich et al., 2007; Jaramillo and Zador, 2011; Niwa et al., 2012a; Bizley et al., 2013; Jaramillo et al., 2014). However, the degree to which interneuronal correlations in A1 reflect behavioral states remains unclear. We therefore measured both $r_{\text {tuning }}$ and $r_{\text {noise }}$ between pairs of A1 neurons recorded from the same electrode during both passive listening and when the animal was engaged in threshold discrimination. We asked whether task engagement affects $r_{\text {noise }}$ between Al neurons. We find that both $r_{\text {tuning }}$ and $r_{\text {noise }}$ are on average positive in A1 and that active engagement reduces average $r_{\text {noise }}$, between 
pairs with positive $r_{\text {tuning, }}$, but not between pairs with negative $r_{\text {tuning. }}$. This effect is optimal for sensory discrimination because $r_{\text {noise }}$ in pairs with negative $r_{\text {tuning }}$ tends to be positive and a reduction would impair population coding. Moreover, we find that task engagement often modulates $\mathrm{r}_{\text {tuning }}$ in individual pairs. Our findings highlight the dynamic nature of A1 population coding (Bathellier et al., 2012) and establish selective $r_{\text {noise }}$ modulation as a mechanism for rapid sensory enhancement.

\section{Materials and Methods}

Subjects. We recorded extracellular activity from right primary auditory cortex (A1) in three adult rhesus macaques (Macaca mulatta; two female, one male), weighing 6-11 kg. All procedures were approved by the University of California, Davis Animal Care and Use Committee and met the requirements of the United States Public Health Service policy on experimental animal care.

Stimuli. We presented unmodulated and sinusoidal amplitude-modulated (AM) broadband noise bursts ( $800 \mathrm{~ms}$ duration) across a range of AM frequencies and depths. For a given recording session, a single AM frequency between 2.5 and $1000 \mathrm{~Hz}$ was used. We selected this AM frequency based on the best modulation frequency (BMF) of the multiunit (MU) activity of the recording site; our methods for determining BMF are described in the Physiology section, below. We varied AM depth from $6 \%$ to $100 \%$. We have previously reported our sound generation methods (O'Connor et al., 2011). Briefly, sound signals were produced using a custom MATLAB program and generated with a D/A converter (Cambridge Electronic Design, model 1401). They were then attenuated (TDT Systems, PA5 and Leader LAT-45), amplified (RadioShack, MPA-200), and passed to a speaker (RadioShack, PA-110; or Optimus, Pro-7AV) positioned 0.8 or $1.5 \mathrm{~m}$ in front of the subject, centered at the interaural midpoint. Sounds were generated at a $100 \mathrm{kHz}$ sampling rate and cosine-ramped at the onset and offset $(5 \mathrm{~ms})$. Intensity was calibrated across all sounds (Bruel and Kjaer model 2231) to $63 \mathrm{~dB}$ at the outer ear.

Task. The task is the same as described by Niwa et al. (2012b, 2013). We recorded extracellular activity during each of two different conditions: (1) task engagement and (2) passive listening. During task engagement (active condition), animals indicated whether a sound is AM in a Go/No Go paradigm. Animals completed a single trial by (1) waiting for a cue light to prompt trial initiation, (2) depressing a lever to initiate a trial, (3) listening to two successive sounds, an "S1" (standard), unmodulated noise burst, and an "S2" (test) stimulus, either unmodulated noise (nontarget) or AM noise (target), (4) indicating detection of target by releasing the lever within $800 \mathrm{~ms}$ after S2 offset or indicating the second sound was a nontarget (unmodulated) by keeping the lever depressed for 800 $\mathrm{ms}$ after S2 offset. S1 and S2 were both $800 \mathrm{~ms}$ and were separated by 400 $\mathrm{ms}$ of silence. Animals were rewarded with liquid (juice or water) for both hits (correctly releasing the lever after target presentation) and correct rejections (correctly withholding lever release after nontarget presentation). Animals were informed of both misses (failure to release lever after targets) and false alarms (inappropriate lever release after nontargets) and received a penalty (15-60 s timeout in which a new trial could not be initiated) for false alarms. Within a recording session, a single AM frequency was used, but depth was varied $(6 \%, 16 \%, 28 \%, 40 \%, 60 \%$, $80 \%$, and $100 \%$ depth). Multiple stimuli (16\%, $28 \%$, and $40 \%$ AM
B
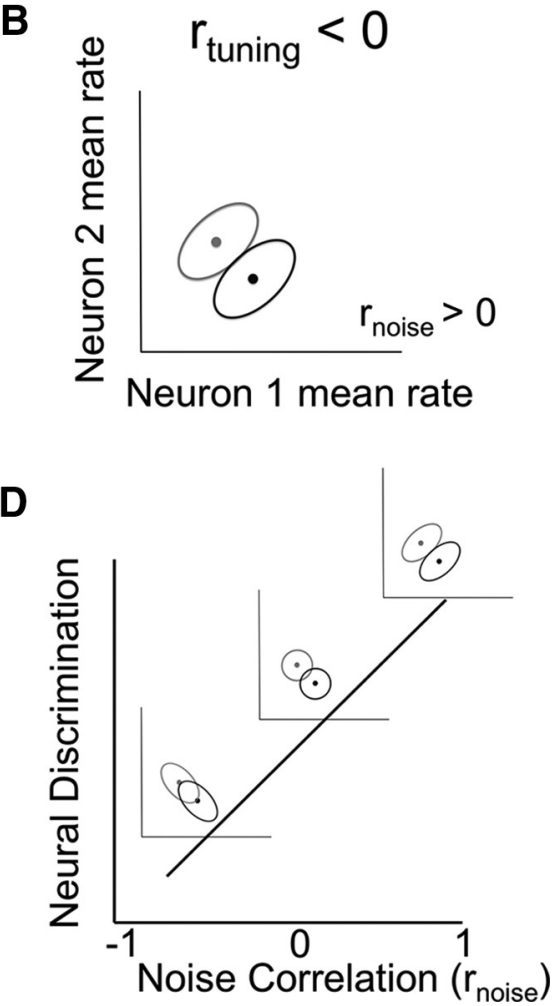

Figure 1. $r_{\text {noise }}$ basic properties. $\boldsymbol{A}, \boldsymbol{B}$, Joint firing rate distributions of hypothetical pairs of neurons to two stimuli. Black represents the joint response to one stimulus. Light gray represents another stimulus. The point within each ellipse represents the mean joint firing rate for that stimulus. The size of the ellipse represents the variance in joint firing rate where one joint rate value pair also exhibits negative $r_{\text {noise }}$ : that is, the ellipses are oriented from top left to bottom right. This joint distribution shape arises wiven trial neuron 1 fires above the mean, neuron 2 is more likely to fire below the mean (and vice versa). The pair in Schematics as in $\boldsymbol{A}$ and $\boldsymbol{B}$. As noise correlation becomes more negative, there is less overlap in the joint firing rates (ellipses in insets), which yields increased neural discrimination. $\boldsymbol{D}$, Effect of $r_{\text {noise }}$ when $r_{\text {tuning }}$ is negative. As $r_{\text {noise }}$ increases, joint firing rates are more separable and neural discrimination improves.

depth) were near animals' AM detection thresholds (O'Connor et al., 2000, 2011; Niwa et al., 2012b).

During passive blocks, animals sat quietly while we presented the same stimuli as in the active condition. Animals received randomly timed liquid rewards. During a recording, animals participated in one passive and one active block, each consisting of $\sim 450$ trials ( $\sim 50$ repetitions per stimulus). For Animals V and W, we counterbalanced which condition, passive or active, came first. For Monkey X, the active condition was always followed by the passive condition. Animals were informed by cue light as to whether they were to respond to sounds (active condition) or not (passive condition).

Physiology. The data presented here are a subset of those presented previously (Niwa et al., 2012b). Briefly, after training on the task, a craniotomy was made over the right parietal cortex. A titanium head post was implanted centrally behind the brow ridge and a CILUX recording cylinder implanted over the craniotomy. During recording, a plastic grid was attached to the cylinder to allow the passage of tungsten microelectrodes (FHC, 1-4 M $\Omega$; Alpha-Omega, 0.5-1 M $\Omega$ ) through a guide tube. The guide tube was used to puncture the dura mater; then the electrode advanced vertically via a hydraulic drive through parietal cortex to A1. During all recordings, animals sat head-restrained in an acoustically transparent chair in a sound-attenuated booth.

Extracellular signals were amplified (AM Systems, model 1800), bandpass filtered between $0.3 \mathrm{~Hz}$ and $10 \mathrm{kHz}$ (Kron-Hite, 3382), then converted to a digital signal at a $50 \mathrm{kHz}$ sampling rate (Cambridge Electronic Design, model 1401). Contributions of single neurons to the signal were 
A

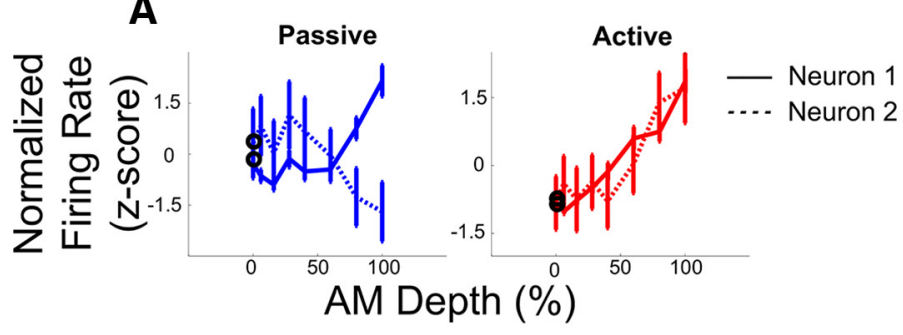

B

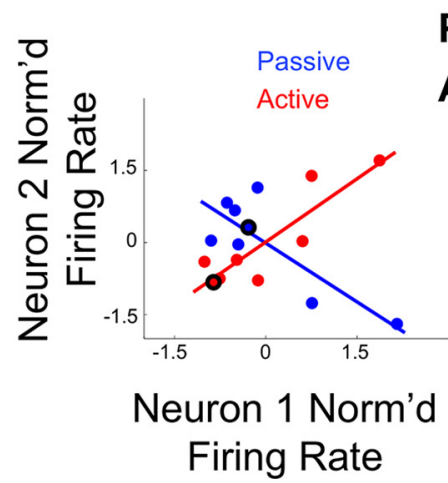

C

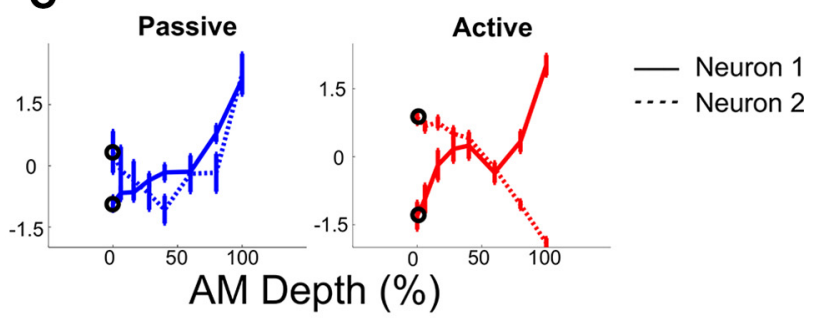

D

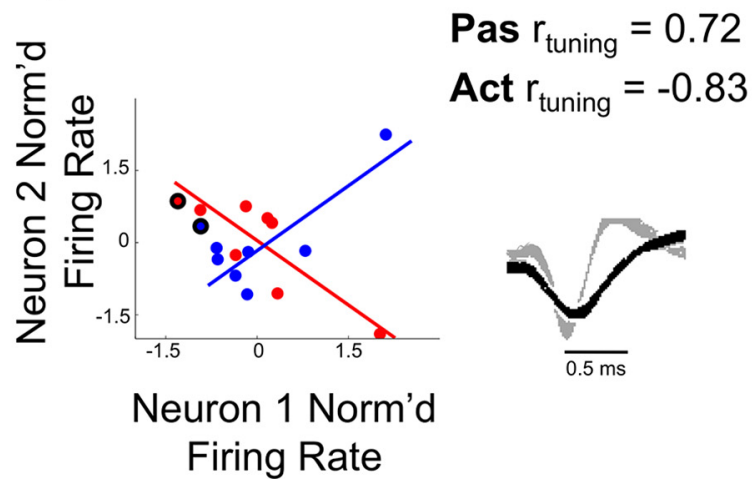

Figure 2. Example pairs exhibit $r_{\text {tuning }}$ shift. $\boldsymbol{A}$, Firing rate versus AM depth functions (rate-depth functions) for two neurons in passive (blue, left) and active (red, right) conditions. Solid line indicates neuron 1. Dotted line indicates neuron 2. Error bars indicate SEM. Black-edged circles represent the nontarget (unmodulated) stimulus. $\boldsymbol{B}$, Characterization of $r_{\text {tuning }}$ by obtaining correlation coefficients ( $r$ ) of paired mean firing rate across stimulus conditions for active (red) and passive (blue) conditions. $\boldsymbol{A}, \boldsymbol{B}$, The example pair exhibits a large shift in $r_{\text {tuning }}(-0.82$, passive to 0.89 , active; $p<0.001$, bootstrap test). Spike waveforms of the two neurons in the pair are shown in black and gray. $\boldsymbol{C}, \boldsymbol{D}$, Same conventions as $\boldsymbol{A}$ and $\boldsymbol{B}$, for another pair that significantly shifts $\mathrm{r}_{\text {tuning }}$ with task condition.

determined offline. We used a cubic spline interpolation algorithm in Spike2 (Cambridge Electronic Design) to create single-unit template waveforms and then match spiking events to those templates. We then used principal components analysis to confirm that events assigned to separate single units formed separable clusters in principal component space. Thresholds for determining spiking activity above background noise were determined visually by the experimenters with the aid of Spike2's automatic trigger-setting algorithm. Spiking activity was generally $4-5$ times the background noise level. Fewer than $0.2 \%$ of spike events assigned to single-neuron clusters fell within a $1 \mathrm{~ms}$ refractory period window. Only recordings in which $>1$ neuron was isolated and held during an entire recording block are analyzed in the present report.

We determined which AM frequency to present during the experiment by finding the BMF of the multi-unit (MU) activity at each recording site. MU activity was defined as any clear spiking activity well above the background noise level of the recording. After an auditory-responsive site was found, we presented $800 \mathrm{~ms}$ AM stimuli across a range of frequencies, all at $100 \%$ depth, as well as $800 \mathrm{~ms}$ unmodulated stimuli. All firing rates were calculated over the entire $800 \mathrm{~ms}$ stimulus period only. Then, we used signal detection analyses (receiver operating characteristic area) to find the AM frequency that the MU activity best discriminated from unmodulated sounds (i.e., the BMF). We calculated both rate-based and temporal measures (spike count and vector strength, respectively) of MU activity; thus, we derived two BMFs at each recording site. Sometimes, the spike count-based BMF (BMFsc) differed from the vector strengthbased BMF (BMFvs). In these cases, we alternated which BMF we used, such that we used the BMFsc whether we used BMFvs the last time the issue arose, and vice versa.

We used stereotactic coordinates to target A1, and we used physiological response properties during recording. In one animal, we anatomically confirmed our recording location (the other two are still serving as research subjects). We measured the pure-tone tuning of recorded neurons and determined their location in A1 based on the tonotopic gradient and the sharpness of their pure-tone tuning relative to neurons recorded in belt regions. Pure-tone tuning was assessed by presenting $100 \mathrm{~ms}$ pure-tones varying across frequency and intensity, with at least three repetitions for each frequency/intensity combination. Stimuli were presented in random order. This allowed us to measure each unit's frequency response area by finding the contour line for frequency/intensity combinations that evoked firing rates at least $2 \mathrm{SD}$ above the spontaneous firing rate (determined in a $75 \mathrm{~ms}$ window before stimulus presentation). Thus, we could measure each unit's characteristic frequency (CF) and sharpness of tuning: bandwidth (BW). In each animal, we mapped CF and BW to determine the topographic distribution of each. Recordings from a region with a high-to-low caudal-to-rostral CF gradient with narrow BW were assigned to A1.

In one animal, we confirmed our stereotactic and physiological assignments by performing postmortem histological analyses. Upon termination of recording, we inserted biotinylated dextran amine to the rostral, middle, and caudal regions of physiologically determined A1 at the border between $\mathrm{A} 1$ and middle-medial belt. The animal was then given an overdose of sodium pentobarbital and perfused using 4\% PFA in $0.1 \mathrm{M}$ phosphate buffer. The brain was removed, blocked, and sliced into 50 $\mu \mathrm{m}$ sections, and slices were stained in alternation with the following: (1) mouse anti-parvalbumin $\rightarrow$ biotinylated horse anti-mouse secondary $\rightarrow$ acetylavidin biotinylated peroxidase complex $(\mathrm{ABC}) \rightarrow$ diamino benzadine (DAB); (2) Nissl substance; (3) Nissl substance $\rightarrow$ ABC $\rightarrow$ DAB. This histology was previously shown by O'Connor et al. (2010), and our physiologically determined A1 borders were validated with anatomical evidence (e.g., dense parvalbumin staining in the area within the biotinylated dextran amine markers).

Data analysis: selection of single neurons for analysis. Neurons can use both rate and temporal codes to represent AM (Liang et al., 2002; Yin et al., 2011; Johnson et al., 2012). Although many neurons display both rate and temporal codes, some do not respond to AM or the carrier by changing firing rate (Yin et al., 2011). In the present analysis, we measured correlations in firing rate both within and across stimuli between Al neurons. Thus, we excluded from analysis any neurons that do not respond to those sounds by changing firing rate. To do so, we tested whether the firing rate in response to any stimulus presented during 
A

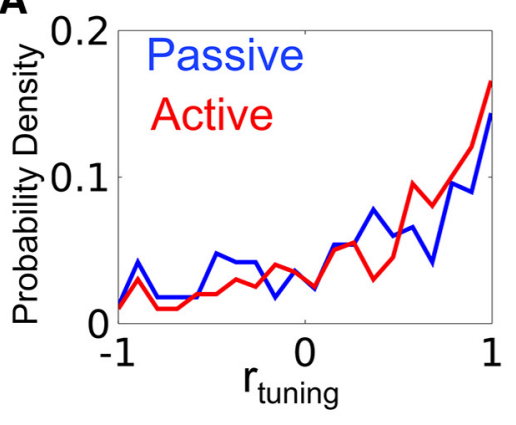

B

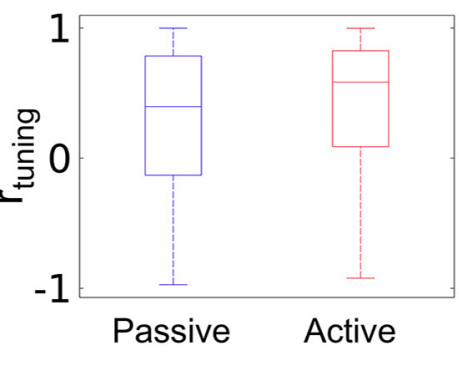

C

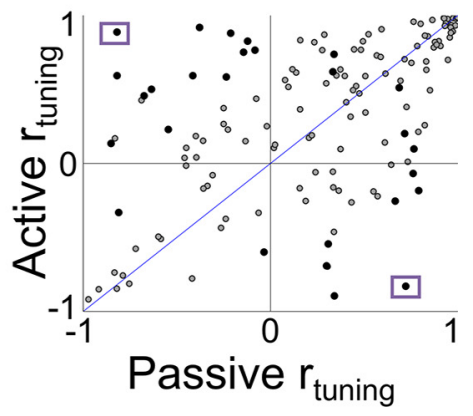

Figure 3. Population $r_{\text {tuning }}$ summary. $A$, Overall distribution of $r_{\text {tuning }}$ across the population in both task conditions. AM $r_{\text {tuning }}$ is mostly strongly positive in $A 1$. $B$, Median (middle line), upper and lower quartiles (bounded by box), and range (whiskers) of $r_{\text {tuning }}$ values in each condition. There is no significant median $r_{\text {tuning }}$ shift between passive and active conditions. $\boldsymbol{C}$, $S_{\text {Scatter of }} r_{\text {tuning }}$ in pairs isolated during both passive and active task conditions and illustrates the degree of $r_{\text {tuning }}$ variability between conditions, although $r_{\text {tuning }}$ is significantly correlated between conditions $\left(r_{(143)}=\right.$ $0.55, p<0.001)$. Pairs that significantly shift $r_{\text {tuning }}(p<0.001$, bootstrap test) between conditions are shown with large black markers. Examples from Figure $2 A-D$ are highlighted with purple boxes.

either task condition was significantly different from the prestimulus baseline firing rate (rank-sum test, $p<0.05$, corrected for multiple comparisons). In the present report, we found 221 pairs of neurons suitable for analysis: 167 pairs in the passive condition, 199 in the active condition, and 145 in both conditions.

Tuning correlation $\left(r_{\text {tuning }}\right)$ and noise correlation $\left(r_{\text {noise }}\right)$. Both $r_{\text {tuning }}$ and $r_{\text {noise }}$ were computed for each pair in each condition. $r_{\text {tuning }}$ is the Pearson correlation between the mean firing rates of each neuron in the pair to the set of 8 stimuli $(0 \%, 6 \%, 16 \%, 28 \%, 405,60 \%, 80 \%$, and $100 \%$ AM) used during a recording. How $r_{\text {noise }}$ was calculated depended on the analysis being performed. When analyzing effects without collapsing across AM depths, $r_{\text {noise }}$ was calculated separately for each of the 8 stimuli, that is, as the Pearson correlation between the trial-by-trial firing rates of each neuron to each of the repetitions of a given stimulus. The minimum number of repetitions of a given stimulus was 27, the maximum was 71 and the average was 50.5 . When collapsing across stimuli to derive a single $r_{\text {noise }}$ measure for a pair, firing rates within each stimulus were $z$-scored, then combined into a single vector of normalized firing rates. Then, the Pearson correlation between these vectors was calculated. To confirm the robustness of our results, we also used nonparametric Spearman correlation to calculate $r_{\text {tuning }}$ and $r_{\text {noise }}$ (data not shown) and none of our major findings changed.

The focus of the present study is the examination of $r_{\text {tuning }}$ and $r_{\text {noise }}$ in A1 during both passive and active task conditions, and the corresponding effects on neural discrimination. Figure 1 displays how $r_{\text {tuning }}$ and $r_{\text {noise }}$ interact to affect neural discrimination. Figure $1, A$ and $B$, each depict two hypothetical neurons' responses to two different stimuli. The firing rate of one neuron is plotted on the $x$-axis, and the other on the $y$-axis (i.e., the plot is the joint firing rate distribution). The distribution is plotted as a dot (mean value) within an ellipse (variance). To the extent that the ellipses of the two joint distributions overlap, that pair of neurons fails to discriminate between those stimuli because joint responses within that overlapping region can arise in response to either stimulus. The pair of neurons in Figure $1 A$ exhibits positive $r_{\text {tuning }}$ and negative $r_{\text {noise }}$ and the pair in Figure $1 B$ exhibits negative $r_{\text {tuning }}$ and positive $r_{\text {noise }}$. The overlap between the joint responses is minimal in each of these cases. Figure $1 C, D$ represents the relationship between $\mathrm{r}_{\text {noise }}$ and neural discrimination for pairs with positive and negative $r_{\text {tuning }}$, respectively. When $\mathrm{r}_{\text {tuning }}$ is positive (Fig. 1, left, C), neural discrimination decreases as $r_{\text {noise }}$ increases. The ellipses in the Figure 1 insets illustrate this effect: given a constant $r_{\text {tuning }}$ value, increases in $r_{\text {noise }}$ yield increased overlap between joint response distributions to the two stimuli, and thus poorer neural discrimination. On the other hand, when $r_{\text {tuning }}$ is negative, neural discrimination increases as $r_{\text {noise }}$ increases. Again, the Figure 1 insets illustrate this effect by showing how the joint response distribution overlap decreases as $r_{\text {noise }}$ increases.

Neural discrimination. The ability of pairs of neurons to discriminate between nontarget and target sounds was assessed using a binomial lo- gistic regression model, similar to that used by Jeanne et al. (2013). Binomial logistic regression is useful for making binary classifications based on a set of variables. In this case, the binary classification is between nontarget and target sounds, and the variables used to make the classification are the firing rates for each of the neurons in the pair. Thus, the model takes as inputs two firing rates and outputs a single classification prediction value between 0 ( $0 \%$ likely to be classified as target) and 1 ( $100 \%$ likely to be classified as target). We fit the model parameters for each stimulus based on data from one-half of the target trials and onehalf of the nontarget trials (even or odd repetitions of each stimulus) and tested classifier performance on a trial-by-trial basis for the remaining one-half of trials. For each trial, the classifier produced a single classification prediction value corresponding to the probability that the two firing rates on that trial were in response to the target stimulus. The percentage of correct classifications for a target stimulus (neural hit rate) was simply the mean prediction classification value. The neural falsealarm rate was the proportion of classifications of nontarget stimuli as targets. Therefore, the percentage of correct classifications for the nontarget stimulus was simply $1-$ (neural false alarm rate). The overall percentage of correct classifications for each pair at each AM depth was the weighted mean of these two values such that:

$$
\begin{aligned}
& \% \text { Correct }=[(\# \text { Target Trials }) *(\text { Neural Hit Rate }) \\
& +(\# \text { Non-Target Trials }) *(1-\text { Neural False Alarm Rate })] / \#
\end{aligned}
$$

Total Trials

We used the MATLAB function 'glmfit' with a logistic link function to implement these analyses. This link function assigns coefficients to joint firing rates using the equation:

$$
\ln (y /(1-y))=b 1(X 1)+b 2(X 2)+a
$$

When fitting the model, $y$ is set to 1 (target stimuli) or 0 (nontarget stimuli). $X 1$ and $X 2$ are the vectors of trial-by-trial firing rates for neuron 1 and neuron 2 , respectively. The 'glmfit' function assigns the coefficients " $b 1$ " and " $b 2$ " and intercept " $a$ " using maximum likelihood estimation. After we fit these parameters using one-half of the available trials as described above, we tested the performance of each pair by using the remaining one-half of trials to derive a prediction classification value for each pair for each trial using 'glmval' in MATLAB.

$r_{\text {noise }}$ simulation. Because there exist multiple aspects of a pair's activity in addition to $r_{\text {noise }}$ that exhibit task-related shifts (e.g., firing rate, Fano factor, and $r_{\text {tuning }}$ ) that could contribute to observed changes in stimulus discrimination, we sought to determine the unique contribution of $r_{\text {noise }}$ To do so, we performed a simulation where we manipulated $r_{\text {noise }}$ while keeping other variables constant. This simulation artificially imposed the passive $r_{\text {noise }}$ values on active recordings. If task-related changes in $r_{\text {noise }}$ 
A

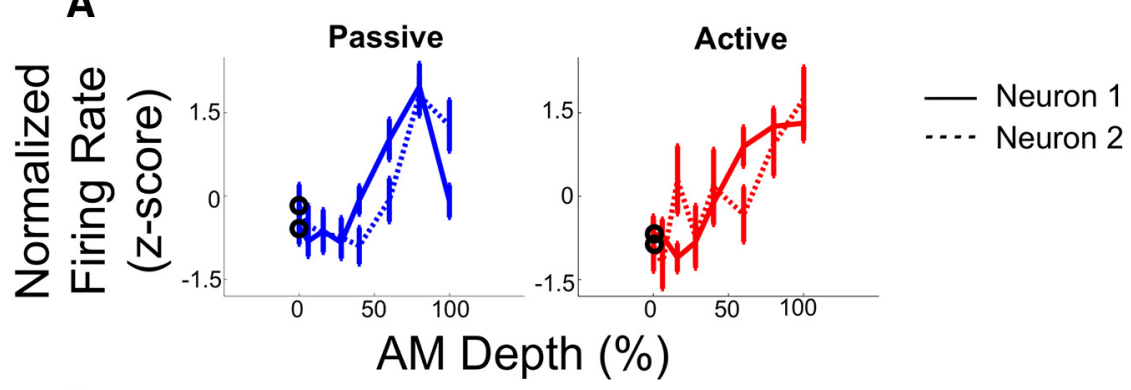

B

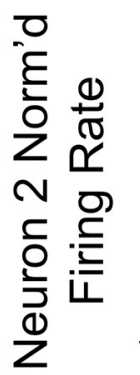

C

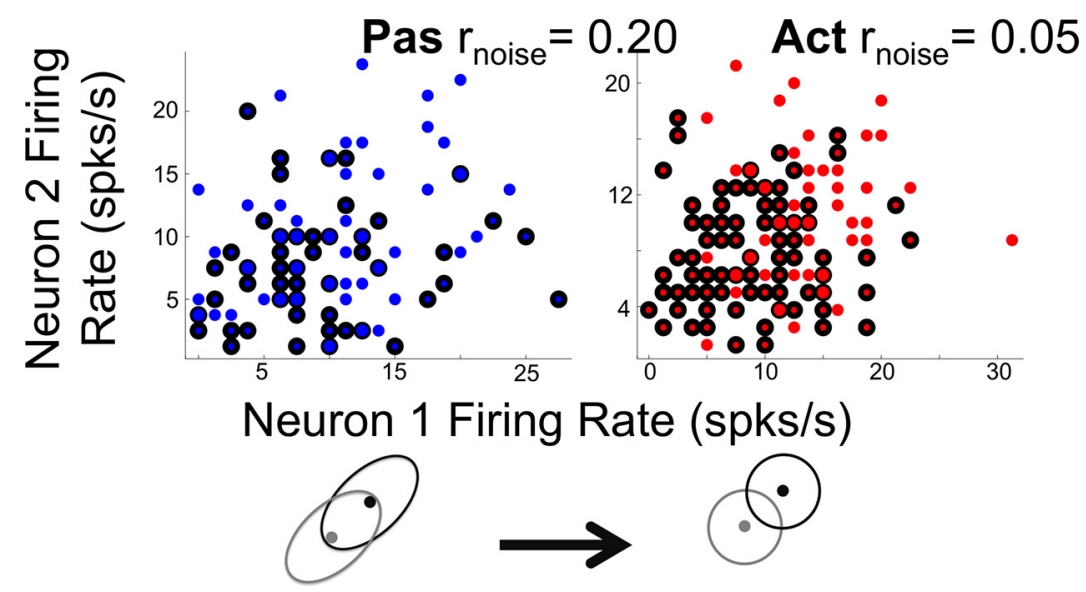

Figure 4. Example pair exhibits reduced $r_{\text {noise }}$ during task engagement. $\boldsymbol{A}$, Rate-depth functions for each neuron in a pair in both passive (blue) and active (red) conditions. Because both neurons exhibit approximately monotonic increasing rate-depth functions, $r_{\text {tuning }}$ is positive $(\boldsymbol{B})$. Also shown in $\boldsymbol{B}$ are the spike waveforms of the two neurons of this pair. $\boldsymbol{C}$, Trial-wise responses to repeated presentations of unmodulated noise (black-edged points) and 100\% AM targets in passive (blue) and active (red) conditions. Each point indicates the joint firing rate in response to a single stimulus presentation. This pair exhibits a decrease in $r_{\text {noise }}$ during active task engagement. The schematics at the bottom represent the relationship to the framework set up in Figure 1. der simulated passive $r_{\text {noise }}$. Any differences in classification performance could thus be attributed to observed shifts in $r_{\text {noise }}$ between the passive and active conditions.

\section{Results}

Task engagement modulates $\mathbf{r}_{\text {tuning }}$ and $\mathbf{r}_{\text {noise }}$

We measured both $r_{\text {tuning }}$ and $r_{\text {noise }}$ between pairs of A1 neurons recorded simultaneously from a single electrode during both passive and active conditions. Intuitively, $r_{\text {tuning }}$ can be thought of as the similarity of tuning between neurons, whereas $r_{\text {noise }}$ can be thought of as the degree to which two neurons' trial-by-trial firing rate variability is correlated. Although we expected $r_{\text {tuning }}$ to exhibit no change between task conditions, we found that a large proportion of pairs exhibited a change in $r_{\text {tuning, with many even shifting }}$ sign between passive and active conditions. Among 145 pairs tested in both conditions, 27 (19\%) exhibit a significant

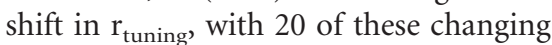
$r_{\text {tuning }} \operatorname{sign}$ (examples in Fig. 2, summary in Fig. 3C). The statistical significance of shifts in $r_{\text {tuning }}$ was assessed via a bootstrap analysis performed on each pair. For this analysis, we estimated confidence intervals for $r_{\text {tuning }}$ in the passive condition and determined whether the active $r_{\text {tuning }}$ was outside of this interval. This analysis was performed as follows: (1) For each neuron in a pair, we resampled (with replacement) trial-by-trial firing rates in response to each of the 8 stimuli in the passive condition. (2) Based on these simulated joint firing rates, a new $r_{\text {tuning }}$ value was calculated. (3) This process was repeated 1000 times for each pair to create a distribution of simulated passive-condition $\mathrm{r}_{\text {tuning }}$ values. Active $\mathrm{r}_{\text {tuning }}$ values that were $<0.1 \%(p<0.001)$ likely to occur via random shifts in passive $r_{\text {tuning }}$ were considered to have significantly shifted.

This effect was surprising to us, given that studies of the behavioral effects on $r_{\text {noise }}$ have not reported $r_{\text {tuning }}$ shifts. Howin the real data contributed to improved stimulus discrimination, then the imposition of passive $r_{\text {noise }}$ on active recordings should decrease neural discrimination. This simulation involved 5 steps for each pair, for each stimulus. (1) For each neuron in the pair, we randomly shuffled the vector of trial-by-trial firing rates in response to the stimulus in the active condition. The result of this shuffling was that firing rate, Fano factor, and $r_{\text {tuning }}$ were unchanged while giving a new $r_{\text {noise }}$ value. (2) Both $r_{\text {noise }}$ and classification performance, as described above, were calculated. (3) Steps 1 and 2 were repeated 1000 times. (4) After 1000 shuffles, we selected the simulated $r_{\text {noise }}$ value that most closely matched the actual passive $r_{\text {noise }}$ value. (5) If the simulated passive $r_{\text {noise }}$ for a given stimulus was not within $\pm 5 \%$ of the actual passive $r_{\text {noise }}$, we excluded that pair/ stimulus from further analysis. After performing steps $1-5$ for each pair at each stimulus, we calculated an average classification performance across all pairs at each stimulus. This allowed us to compare classification performance in the active condition with classification performance un- ever, it is worth noting that Winkowski et al. (2013) found rapid shifts in $r_{\text {tuning }}$ with frontal cortex microstimulation. Some neurons had exceptionally large shifts in $r_{\text {tuning }}$ (Fig. $2 A-D$ ). In Figure $2 A$, two neurons' responses as a function of AM depth are shown. In the passive condition, neuron 1's firing rate (solid line) increases with increasing AM depth (increasing rate-depth function), whereas neuron 2's firing rate (dashed line) decreases with increasing AM depth (decreasing rate-depth function). Thus, the pair exhibits negative $r_{\text {tuning }}$. In the active condition, however, both neurons exhibit increasing rate-depth functions, leading to positive $r_{\text {tuning }}$. Figure $2 B$ plots the joint mean firing rate distribution for each of the eight stimuli in active and passive conditions (black-edged circles represent responses to unmodulated noise). The large shift from -0.82 (passive) to 0.89 (active) is 
clear by observing the least-squares lines slopes. In the example shown in Figure $2 C, D$, both neurons exhibit increasing rate-depth functions in the passive condition, but in the active condition, the neurons have opposite rate-depth functions. Figure $2 D$, like Figure $2 B$, shows each neuron's mean firing rates in each condition and illustrates this pair's large shift in $r_{\text {tuning }}$.

Across the entire population, however, we observed no significant change in median $\mathrm{r}_{\text {tuning }}$ (Fig. $3 A, B$ ). However, even though there may be no statistically significant shifts in median $r_{\text {tuning, shifts in }}$ $\mathrm{r}_{\text {tuning }}$ between individual pairs can critically impact population coding. Although Figure $3 B$ shows no significant shift in population median $r_{\text {tuning, }}$ individual pairs still do exhibit shifts. Figure $2 B, D$ provides examples of this and the magnitudes of their $r_{\text {tuning }}$ shifts are highlighted (purple boxes) in Figure 3C. Because many of our analyzable pairs exhibited shifts in $\mathrm{r}_{\text {tuning }}$ sign (Fig. 3C, top left, bottom right), we analyzed $r_{\text {tuning }} / r_{\text {noise }}$ relationships separately in each task condition. Thus, when we analyzed $r_{\text {noise }}$ effects based on $r_{\text {tuning }}$ sign, we grouped each pair's $r_{\text {noise }}$ value independently in each task condition based on $r_{\text {tuning }}$ from that condition only rather than averaging $r_{\text {tuning }}$ across conditions.

We hypothesized that task engagement enhances population coding by selectively reducing average $r_{\text {noise }}$ for pairs with positive, but not negative, $r_{\text {tuning }}$. Two representative examples demonstrate this. One example shows the $r_{\text {noise }}$ reduction for a pair of neurons with positive $r_{\text {tuning }}$ (Fig. 4), and one example (Fig. 5) shows the $r_{\text {noise }}$ increase for a pair of neurons with negative $r_{\text {tuning }}$. For the pair with positive $\mathrm{r}_{\text {tuning }}$ both neurons have increasing ratedepth functions (Fig. 4A), and active engagement leads to a decrease in $r_{\text {noise }}$ in this pair (Fig. 4C). $r_{\text {noise }}$ reduction was the more common effect in our data, although many pairs increase $r_{\text {noise }}$ with task engagement ( 85 of 145 pairs decrease $r_{\text {noise }}$ with task engagement, $p=0.04, \chi^{2}$ test). For the neuron pair with negative $r_{\text {tuning }}$ active engagement leads to an increase in $r_{\text {noise }}$ (Fig. 5C). In Figure $4 C$, we show scatter plots for the unmodulated sound (black-edged circles) and 100\% modulation (no-edged circles). The schematic ellipses are meant to demonstrate how the distributions go from more oblong (positive $r_{\text {noise }}$, left), to more circular $\left(r_{\text {noise }}\right.$ close to 0 ). Figure 5 shows an example with negative $r_{\text {tuning, }}$, where $r_{\text {noise }}$ increases during the active condition. We assessed the significance of shifts in $r_{\text {noise }}$ for individual pairs by conducting analysis of covariance (ANCOVA) for each pair using MATLAB's 'aoctool' function. In essence, this analysis tests for differences in the relationship between two variables (e.g., $\mathrm{r}_{\text {noise }}$ ) between two conditions (e.g., task engagement). A total of 28 of 145 pairs tested in
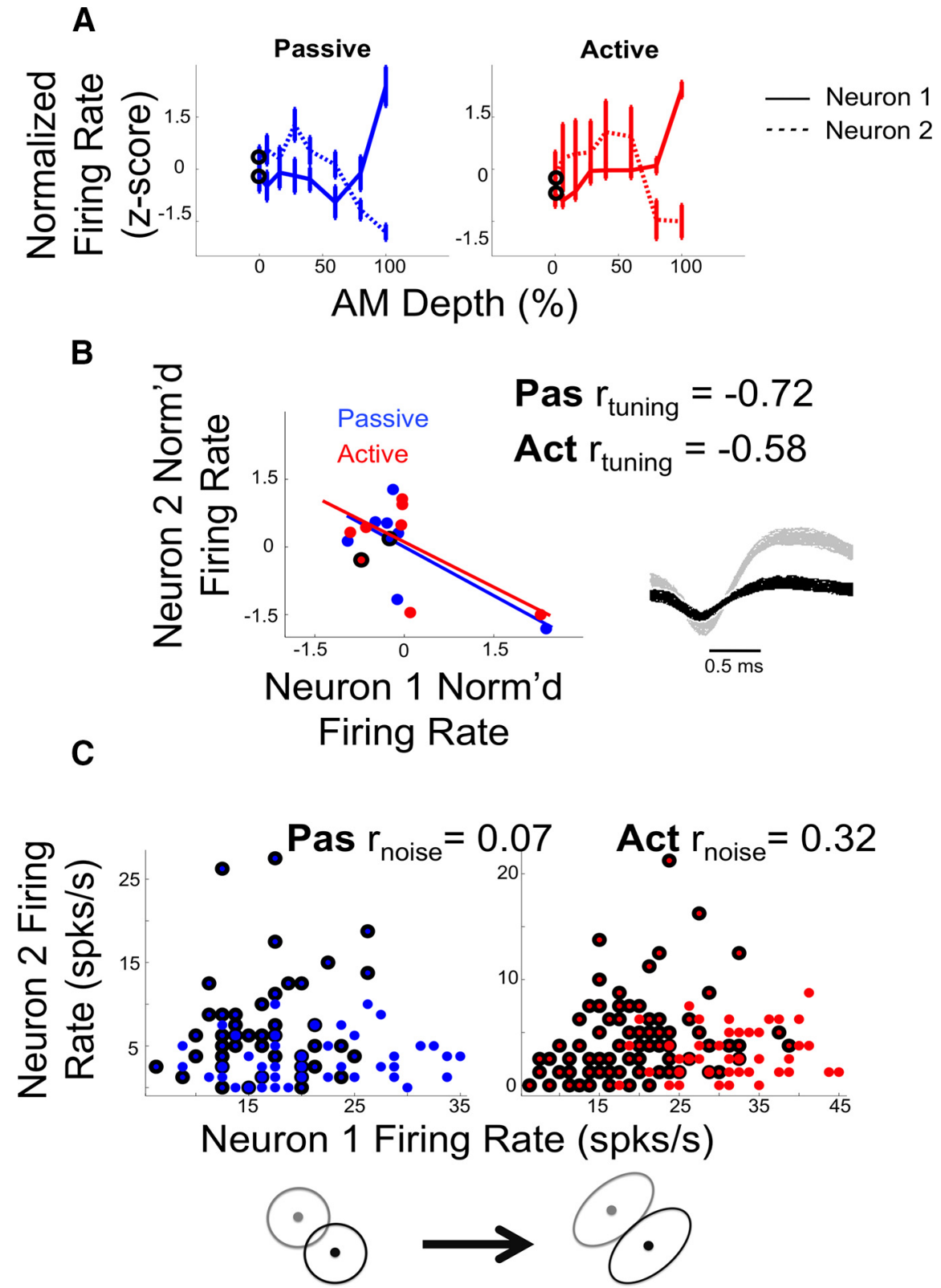

Figure 5. Example pair exhibits increased $r_{\text {noise }}$ during task engagement. Conventions as in Figure 3. In this example, the two neurons exhibit approximately monotonic rate-depth functions, with one increasing and the other decreasing its rate as AM depth increases $(\boldsymbol{A})$. Thus $\mathrm{r}_{\text {tuning }}$ is negative $(\boldsymbol{B})$. As in Figures 2 and 4 , we show the spike waveforms of the neurons of the pair. The spike represented by the waveform in black spiked well above background noise level, but the scaling of the image due to the size of the gray waveform obscures this. This pair exhibits an increase in $r_{\text {noise }}$ during active task engagement $(\boldsymbol{C})$.

both conditions exhibit a significant decrease, whereas 16 exhibit a significant increase in $r_{\text {noise }}(p<0.05$, corrected using false discovery rate) (Benjamini and Yekutieli, 2001). We further asked whether $r_{\text {tuning }}$ sign could predict the direction of significant $r_{\text {noise }}$ shifts in individual pairs. Grouping pairs by either passive or active $r_{\text {tuning, }}$, we find that significant $r_{\text {noise }}$ shifts are approximately evenly distributed for pairs with negative $r_{\text {tuning }}$ (grouped by passive $r_{\text {tuning }}, 8$ of 18 decrease; grouped by active $r_{\text {tuning }}, 7$ of 16 decrease). Significant $r_{\text {noise }}$ shifts for pairs with positive $r_{\text {tuning }}$ seem more likely to decrease than increase (grouped by passive $\mathrm{r}_{\text {tuning }}$, 18 of 26 decrease; grouped by active $\mathrm{r}_{\text {tuning }} 19$ of 28 decrease). A $\chi^{2}$ test reveals this effect to be insignificant, although the direction of the trend is consistent with a selective $r_{\text {noise }}$ decrease that depends on $r_{\text {tuning. }}$ We 
A

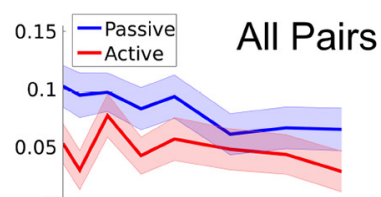

0 .

0

C

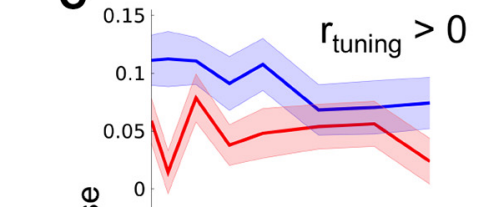

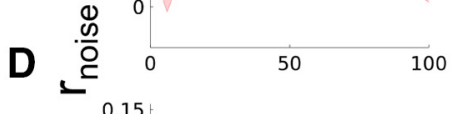

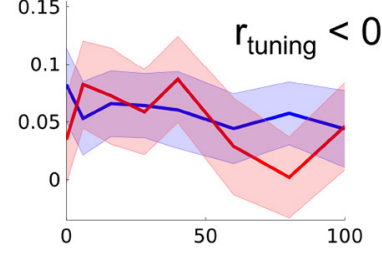

AM Depth (\%)

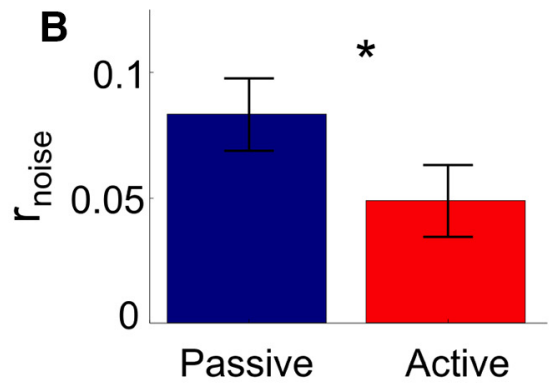

E

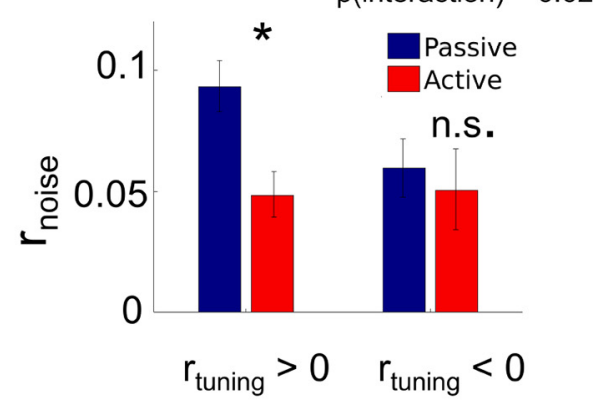

Figure 6. Summary of $r_{\text {noise }}$ effects. $\boldsymbol{A}, r_{\text {noise }}$ across all pairs, by AM depth. $r_{\text {noise }}$ decreases significantly as AM depth increases (ANOVA; $p<0.001) . B, r_{\text {noise }}$ collapsed across all stimuli. Active engagement significantly decreases $r_{\text {noise }}$ across the entire population (ANOVA; $p<0.001$ ). C, D, Same as in $\boldsymbol{A}$, grouped by $r_{\text {tuning }}$ sign. $\boldsymbol{E}$, $r_{\text {noise }}$ collapsed across all stimuli, grouped by $r_{\text {tuning }}$ sign. Active engagement significantly reduces $r_{\text {noise }}$ only for pairs with positive $r_{\text {tuning }}$ (ANOVA; $p_{\text {(interaction) }}=0.02$ ). ${ }^{*} p<0.05 ; n$.s., Not significant.

summarize $r_{\text {noise }}$ effects across all pairs (167 passive and 199 active) in Figure 6.

We see a global decrease in $r_{\text {noise }}$ with task engagement (ANOVA, $p<0.001$ ), as well as a significant decrease in $r_{\text {noise }}$ with increasing AM depth (ANOVA, $p<0.001$ ) (Fig. 6A). Here $r_{\text {noise }}$ is plotted as a function of AM depth. When analyzing the effect of task engagement on $r_{\text {noise }}$ at each AM depth separately, we find the effect is only significant at 6\% AM depth $(p<0.05)$, as assessed by a rank-sum test, corrected for multiple comparisons using false discovery rate (Benjamini and Yekutieli, 2001). When collapsing across all depths, we find that $r_{\text {noise }}$ decreases from a mean value of 0.0812 in the passive condition to a mean value of 0.0472 in the active condition. The finding that task engagement reduces $r_{\text {noise }}$ is consistent with previous reports that $r_{\text {noise }}$ decreases globally as sensory demands increase. However, because reducing $r_{\text {noise }}$ can impair population coding when $r_{\text {tuning }}$ is negative, we analyzed the interaction between task condition and $r_{\text {tuning }}$ on $r_{\text {noise }}$ by including $r_{\text {tuning }}$ in the model. Figure $6 C, D$ shows the effect of task engagement on $r_{\text {noise }}$ for both positive and negative $r_{\text {tuning, }}$, respectively, for each tested stimulus. Because $r_{\text {tuning }}$ sign often changes between conditions, we treated the $r_{\text {noise }}$ distributions for positive and negative $r_{\text {tuning }}$ sign as independent between conditions in our analyses. Using a $2 \times 2$ ANOVA (task condition $\times \mathrm{r}_{\text {tuning }}$ sign; 'aov' function in $\mathrm{R}$ ), we see a global decrease in $r_{\text {noise }}$ for positive $r_{\text {tuning }}$ pairs $(p<0.001$; Fig. $6 C, E)$, but no effect on $r_{\text {noise }}$ for negative $r_{\text {tuning }}$ pairs $(p=0.53$; Fig. $6 D, E)$. Moreover, our analyses reveal a significant interaction effect $(p=0.02)$ wherein task engagement reduces $r_{\text {noise }}$ only for positive $r_{\text {tuning }}$ pairs. Figure $6 E$ illustrates how the effect of task condition on $r_{\text {noise }}$ depends on $r_{\text {tuning }}$ sign, collapsed across tested AM depths.

Figure $7 \mathrm{~A}$ illustrates the overall effect of task engagement on pairs of neurons' ability to discriminate target (AM) from nontarget stimuli (unmodulated noise). We used a binomial logistic regression (described in detail in Materials and Methods) to classify joint firing rates on each trial as either target or nontarget sounds. In both conditions, classifier performance increases with increasing AM depth (ANOVA, $p<0.0001$; 'aov' function in R). Moreover, active engagement increases pairs' performance at each depth relative to passive listening (Fig. $7 A)$. Statistical significance of this effect was determined using ANOVA with pairwise post hoc tests at each depth (Tukey's HSD, $p<0.05$; 'TukeyHSD' function in $\mathrm{R})$. Because we have previously shown that task engagement increases single A1 neuron's AM sensitivity based on firing rate alone (Niwa et al., 2012b), we sought to quantify to what extent the observed improvement in sensitivity for pairs could be uniquely accounted for by changes in $r_{\text {noise }}$. We did so by imposing passive $r_{\text {noise }}$ values on the joint firing rate distributions in the active condition. To do so, we repeatedly shuffled the trial order of active recordings (1000 repetitions) to obtain new $r_{\text {noise }}$ values. Of these 1000 simulated $r_{\text {noise }}$ values, we selected for further analysis the shuffle that most closely approximated the passive $r_{\text {noise }}$ value (hereafter known as the "simulated passive $r_{\text {noise") }}$ ) and calculated classifier performance for that shuffle. Thus, since shuf-

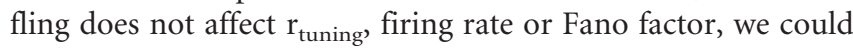
directly assess how differences between passive and active $r_{\text {noise }}$ uniquely contribute to changes in classifier performance. The simulated passive $r_{\text {noise }}$ values are shown in Figure $7 B-D$. It is important to note that simulated passive $r_{\text {noise }}$ is virtually equal to passive $r_{\text {noise }}(0.0074 \%$ different on average); but as we have graphed them, simulated passive $r_{\text {noise }}$ appears to only approximate the true passive $r_{\text {noise. }}$ The ostensible difference arises from the fact that we group simulated passive $r_{\text {noise }}$ by active $r_{\text {tuning }}$, while we group true passive $r_{\text {noise }}$ by passive $r_{\text {tuning. }}$. $r_{\text {tuning }}$ often shifts between conditions. Thus, for a given pair, simulated passive $r_{\text {noise }}$ may be identical to the true passive $r_{\text {noise }}$ value but will be grouped separately if $r_{\text {tuning }}$ shifts for that pair. We group simulated passive $r_{\text {noise }}$ by active $r_{\text {tuning }}$ because we derive simulated passive $r_{\text {noise }}$ from active recordings. If we grouped simulated passive $r_{\text {noise }}$ by passive $r_{\text {tuning }}$, or if $r_{\text {tuning }}$ was constant between conditions, the simulated passive and true passive $r_{\text {noise }}$ graphs would appear virtually identical in Figure $7 B-D$.

Figure $7 E, F$ represents the average difference between classifier performance for active recordings with active $r_{\text {noise }}$ and active recordings with simulated passive $r_{\text {noise }}$. Values that significantly deviate from 0 (rank-sum test, $p<0.05$, corrected) indicate that shifts in $r_{\text {noise }}$ due to active engagement provide a unique contribution to observed shifts in classifier performance. Because we find that $r_{\text {noise }}$ decreases with active engagement for pairs with positive $r_{\text {tuning }}$ but stays the same for pairs with negative $r_{\text {tuning, }}$, we 
predicted that the $r_{\text {noise }}$ should contribute to shifts in classifier performance only for pairs with positive $r_{\text {tuning }}$. Consistent with this prediction, we find that classifier performance is higher on average with active $r_{\text {noise }}$ than with simulated passive $r_{\text {noise }}$, but only for pairs with positive $r_{\text {tuning }}$. This effect is significant only at AM depths near or below psychophysical threshold (6\%-40\% AM), which may point to a selective role for $r_{\text {noise }}$ reduction during especially difficult trials.

\section{Discussion}

\section{Summary}

We show that actively discriminating between AM and unmodulated sounds reduces $r_{\text {noise }}$ between pairs of similarly tuned (positive $r_{\text {tuning }}$ ) A1 neurons while leaving $r_{\text {noise }}$ unaffected for pairs with dissimilar tuning (negative $r_{\text {tuning }}$ ). Because decreases in $r_{\text {noise }}$ should enhance population coding only when $r_{\text {tuning }}$ is positive, this result suggests that sensory systems can selectively target specific subpopulations within larger neural networks to rapidly and dynamically gate the transmission of sensory information. Although multiple studies have shown that increasing sensory demands lead to rapid, global decreases in $r_{\text {noise }}$, to our knowledge this finding constitutes the first report of a dynamic, $r_{\text {tuning }}$ specific reduction in $r_{\text {noise }}$. Moreover, in contrast to previous studies that assume $r_{\text {tuning }}$ is constant, we directly measured $r_{\text {tuning }}$ in multiple behavioral conditions. Because $r_{\text {tuning }}$ changes with behavioral state, it will be important to account for possible $r_{\text {tuning }}$ changes in future studies. Although the present analyses focus only on the neural coding of AM, the effect we observe wherein deleterious $r_{\text {noise }}$ is reduced and beneficial $r_{\text {noise }}$ is unaffected during task engagement could generalize to any stimulus feature encoded by firing rate.

\section{Basic properties of neural correlations in auditory cortex (AC)}

Our report of an average bias for similar tuning in A1 neuron pairs agrees with other reports that neighboring neurons in AC tend to exhibit similar response properties (Rothschild et al., 2010; Issa et al., 2014). It is worth noting that our observed $r_{\text {tuning }}$ distribution differs from that reported by both Rothschild et al. (2010) and Winkowski et al. (2013). Namely, our median $r_{\text {tuning }}$ values ( 0.49 , active; 0.36 passive) are larger than those reported by Winkowski et al. (2013) (mean $\mathrm{r}_{\text {tuning }}=0.10$ ) or Rothschild et al. (2010) ( mean $r_{\text {tuning }}=0.08$ ). This could be explained by several factors. First, each used tonal stimuli (Winkowski et al., 2013; AM tones) (Rothschild et al., 2010; 50 ms tone-pips), where cells with different pure-tone best frequencies should be less correlated. Second, they recorded over distances of cortex, whereas we only recorded very close neighbors. Third, there could be species dif-
B

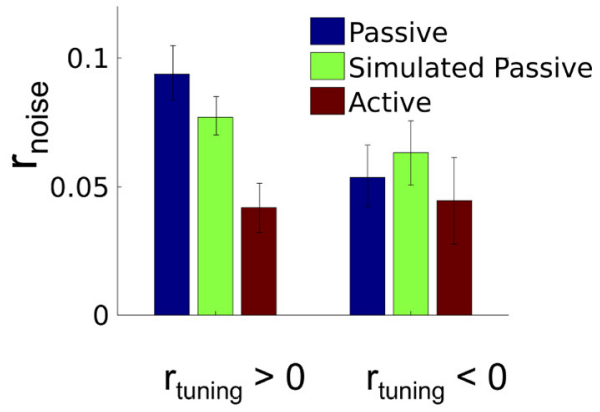

D
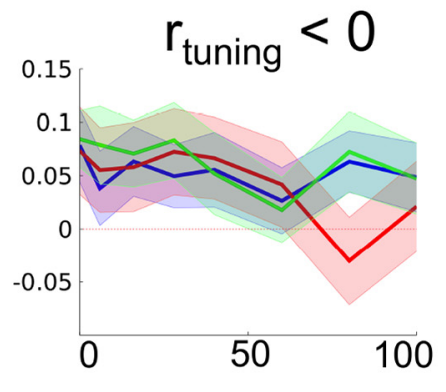

$\mathbf{F}$

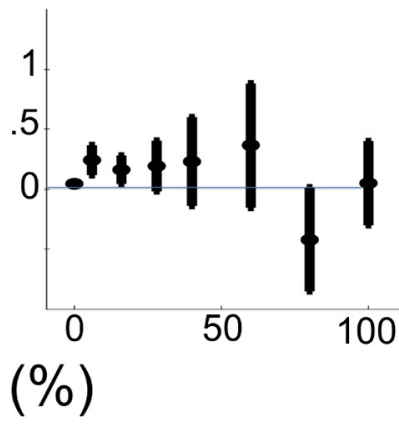

AM Depth (\%)

Figure 7. $\quad r_{\text {noise }}$ effects enhance neural discrimination. $A$, Results of classifier model. At each AM depth, pairs' neural discrimination is better on average during the active (red) than passive (blue) condition (rank-sum test, $p<0.05$ corrected for multiple comparisons). Error bars indicate SEM. Simulated passive $r_{\text {noise }}$ values are shown in $\boldsymbol{B}$ (collapsed across all stimuli), $\boldsymbol{C}$ (positive $r_{\text {tuning }}$ ), and $\boldsymbol{D}$ (negative $r_{\text {tuning }}$ ). $\boldsymbol{E}, \boldsymbol{F}$, The unique contribution of $r_{\text {noise }}$ on task-related increases in neural discrimination $(\boldsymbol{A})$. lated passive $r_{\text {noise. }}$. Any value $>0$ indicates that active $r_{\text {noise }}$ increases classifier performance above and beyond the effect of other ables, such as firing rate. Effect of $r_{\text {noise }}$ on neural discrimination is significant at the four lowest AM depths (6\%, 16\%, 28\%, and 40\%) for $\mathrm{r}_{\text {tuning }}>0$ and did not reach significance at any depth for $\mathrm{r}_{\text {tuning }}<0$ (rank-sum test, $p<0.05$ corrected). ${ }^{*} p<0.05$.

ferences. Further studies on neural correlations in AC will benefit from testing multiple acoustic features with different recording techniques to glean a clearer picture of network-level feature processing in AC.

Our average $r_{\text {noise }}$ values ( 0.08 passive, and 0.05 active), on the other hand, generally agree with those reported by others (i.e., they are small and positive). Those mentioned above also reported small, slightly positive, $r_{\text {noise }}$ values $(0.18$, Rothschild et al., 2010; no value given for Winkowski et al., 2013). Issa and Wang (2013) also report average $r_{\text {noise }}$ values between 0.08 and 0.20 . Moreover, this result agrees with reports across sensory systems (for review, see Cohen and Kohn, 2011).

\section{Behavioral modulation of $\mathbf{r}_{\text {noise }}$ in visual and AC}

Although the majority of reports on the effect of attention on $\mathrm{r}_{\text {noise }}$ are in visual cortex, we provide the first report of attention- 
related effects on auditory cortical $r_{\text {noise }}$. Our major finding that behavior selectively reduces $r_{\text {noise }}$ based on $r_{\text {tuning }}$ has not been reported in visual cortex, at any level of the system. Instead, most have reported that the shift from an inattentive to attentive state leads to global reductions in $r_{\text {noise }}$, regardless of $r_{\text {tuning }}$ (Cohen and Maunsell, 2009). However, Cohen and Newsome (2008) have previously reported that pairs of neurons in area MT exhibit task-related shifts in $r_{\text {noise }}$ when animals detect dot motion relative to when they fixate, and these shifts depend on both the tuning similarity between the neurons and whether each neuron contributed to the same, or different, perceptual decisions. Namely, they found that pairs most often decreased $r_{\text {noise }}$ during behavior, but when pairs had very dissimilar tuning (which would correspond to very negative $r_{\text {tuning }}$ ), $r_{\text {noise }}$ increased during behavior, but only if the neurons of the pair contributed to opposite dot motion decisions. During another task condition in which both neurons contributed to the same dot motion decision, they observed a decrease in $r_{\text {noise }}$. Although We did not

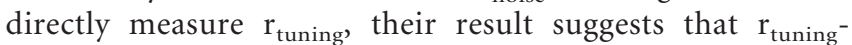
dependent shifts in $r_{\text {noise }}$ are present in visual cortex as well as AC. However, a dearth of studies in which $r_{\text {tuning }}$ is directly measured contributes to an incomplete picture of how behavior affects $r_{\text {noise }}$.

\section{Behavioral modulation of $\mathbf{r}_{\text {tuning }}$}

Here we provide perhaps the first report of behavioral shifts in $r_{\text {tuning. }}$. Although we observe no shift in the median $r_{\text {tuning }}$ value between task conditions, we observe that pairs commonly exhibit shifts in the sign of $\mathrm{r}_{\text {tuning }}$ in the transition between passive listening and active sound detection. However, although others have not reported on this phenomenon, we think it is likely that behavioral variables commonly shift $r_{\text {tuning, }}$, across sensory cortical fields. Attention has been shown to modulate single neuron tuning in $\mathrm{AC}$ and $\mathrm{VC}$ not just via gain modulation, but also by shifts in receptive fields (Fritz et al., 2003; David et al., 2008). Given two single neurons' receptive fields shifting in response to task demands, it follows that $r_{\text {tuning }}$ can often change, sometimes dramatically. Thus, our finding that attention can shift $r_{\text {tuning }}$ values is supported by changes in tuning of single neurons. Given that the effect of $r_{\text {noise }}$ depends on the sign of $r_{\text {tuning, }}$, it will be worthwhile for future studies to investigate how attention affects each of these measures.

\section{Issues with global $\mathbf{r}_{\text {noise }}$ decreases}

Theoretical studies have established that positive $r_{\text {noise }}$ can aid in neural discrimination (Oram et al., 1998; Averbeck et al., 2006; Ecker et al., 2011). Empirical studies have affirmed these ideas. Romo et al. (2003) observed that positive $r_{\text {noise }}$ reduces neural discrimination thresholds for pairs with opposite tuning (which would yield a negative $r_{\text {tuning }}$ value). They recorded pairs of S2 neurons, a cortical field in which single neurons commonly exhibit either increasing or decreasing firing rate functions in response to increasing tactile vibration frequency. Similarly, neurons in A1 (and more so in middle lateral belt) exhibit this "dual coding" scheme for AM depth (Niwa et al., 2013). A potential goal of future research will be to characterize differences in the behavioral effects of $r_{\text {noise }}$ for populations with dual coding relative to those with Gaussian feature tuning, as different mechanisms may be at play in shifting $r_{\text {noise }}$. Jeanne et al. (2013) have also reported that pairs with negative $r_{\text {tuning }}$ benefit from positive $r_{\text {noise }}$. They trained starlings to map specific behavioral responses to specific starling vocalization. Recording from the caudolateral mesopallium (CLM) they found that learning not only reduced $r_{\text {noise }}$ when $r_{\text {tuning }}$ is positive but increased $r_{\text {noise }}$ when $r_{\text {tuning }}$ is negative. The stimuli used in their study (starling vocalizations) are considerably more complex than those used here or by Romo et al. (2003). CLM has been hypothesized to specialize at representing learned vocalization stimuli (Gentner and Margoliash, 2003; Gill et al., 2008). It may be that Jeanne et al. (2013) observed a more powerful effect in CLM than we do in A1 because, although CLM is specialized for learned vocalizations, AM is one of many sound features to which A1 neurons respond (deCharms, 1998; Wang et al., 2005; Chambers et al., 2014). Future studies may manipulate stimulus conditions orthogonally to behavioral variables while recording from neural populations to build a more complete picture of the functional characteristics of neural correlation structure.

\section{References}

Abbott LF, Dayan P (1999) The effect of correlated variability on the accuracy of a population code. Neural Comput 11:91-101. CrossRef Medline

Averbeck BB, Latham PE, Pouget A (2006) Neural correlations, population coding and computation. Nat Rev Neurosci 7:358-366. CrossRef Medline

Bathellier B, Ushakova L, Rumpel S (2012) Discrete neocortical dynamics predict behavioral categorization of sounds. Neuron 76:435-449. CrossRef Medline

Benjamini Y, Yekutieli D (2001) The control of the false discovery rate in multiple testing under dependency. Ann Stat 29:1165-1188. CrossRef

Bizley JK, Walker KM, Nodal FR, King AJ, Schnupp JW (2013) Auditory cortex represents both pitch judgments and the corresponding acoustic cues. Curr Biol 23:620-625. CrossRef Medline

Chambers AR, Hancock KE, Sen K, Polley DB (2014) Online stimulus optimization rapidly reveals multidimensional selectivity in auditory cortical neurons. J Neurosci 34:8963-8975. CrossRef Medline

Cohen MR, Kohn A (2011) Measuring and interpreting neuronal correlations. Nat Neurosci 14:811-819. CrossRef Medline

Cohen MR, Maunsell JH (2009) Attention improves performance primarily by reducing interneuronal correlations. Nat Neurosci 12:1594-1600. CrossRef Medline

Cohen MR, Newsome WT (2008) Context-dependent changes in functional circuitry in visual area MT. Neuron 60:162-173. CrossRef Medline

David SV, Hayden BY, Mazer JA, Gallant JL (2008) Attention to stimulus features shifts spectral tuning of V4 neurons during natural vision. Neuron 59:509-521. CrossRef Medline

deCharms RC, Blake DT, Merzenich MM (1998) Optimizing sound features for cortical neurons. Science 280:1439-1443. CrossRef Medline

Ecker AS, Berens P, Tolias AS, Bethge M (2011) The effect of noise correlations in populations of diversely tuned neurons. J Neurosci 31:1427214283. CrossRef Medline

Ecker AS, Berens P, Cotton RJ, Subramaniyan M, Denfield GH, Cadwell CR, Smirnakis SM, Bethge M, Tolias AS (2014) State dependence of noise correlations in macaque primary visual cortex. Neuron 82:235-248. CrossRef Medline

Fritz J, Shamma S, Elhilali M, Klein D (2003) Rapid task-related plasticity of spectrotemporal receptive fields in primary auditory cortex. Nat Neurosci 6:1216-1223. CrossRef Medline

Gentner TQ, Margoliash D (2003) Neuronal populations and single cells representing learned auditory objects. Nature 424:669-674. CrossRef Medline

Gill P, Woolley SM, Fremouw T, Theunissen FE (2008) What's that sound? Auditory area CLM encodes stimulus surprise, not intensity or intensity changes. J Neurophysiol 99:2809-2820. CrossRef Medline

Gu Y, Liu S, Fetsch CR, Yang Y, Fok S, Sunkara A, DeAngelis GC, Angelaki DE (2011) Perceptual learning reduces interneuronal correlations in macaque visual cortex. Neuron 71:750-761. CrossRef Medline

Herrero JL, Gieselmann MA, Sanayei M, Thiele A (2013) Attention-induced variance and noise correlation reduction in macaque $\mathrm{V} 1$ is mediated by NMDA receptors. Neuron 78:729-739. CrossRef Medline

Issa EB, Wang X (2013) Increased neural correlations in primate auditory cortex during slow-wave sleep. J Neurophysiol 109:2732-2738. CrossRef Medline

Issa JB, Haeffele BD, Agarwal A, Bergles DE, Young ED, Yue DT (2014) 
Multiscale optical $\mathrm{Ca}^{2+}$ imaging of tonal organization in mouse auditory cortex. Neuron 83:944-959. CrossRef Medline

Jaramillo S, Zador AM (2011) The auditory cortex mediates the perceptual effects of acoustic temporal expectation. Nat Neurosci 14:246-251. CrossRef Medline

Jaramillo S, Borges K, Zador AM (2014) Auditory thalamus and auditory cortex are equally modulated by context during flexible categorization of sounds. J Neurosci 34:5291-5301. CrossRef Medline

Jeanne JM, Sharpee TO, Gentner TQ (2013) Associative learning enhances population coding by inverting interneuronal correlation patterns. Neuron 78:352-363. CrossRef Medline

Johnson JS, Yin P, O'Connor KN, Sutter ML (2012) Ability of primary auditory cortical neurons to detect amplitude modulation with rate and temporal codes: neurometric analysis. J Neurophysiol 107:3325-3341. CrossRef Medline

Liang L, Lu T, Wang X (2002) Neural representations of sinusoidal amplitude and frequency modulations in the primary auditory cortex of awake primates. J Neurophysiol 87:2237-2261. Medline

Mitchell JF, Sundberg KA, Reynolds JH (2009) Spatial attention decorrelates intrinsic activity fluctuations in macaque area V4. Neuron 63:879888. CrossRef Medline

Nirenberg S, Latham PE (2003) Decoding neuronal spike trains: how important are correlations? Proc Natl Acad Sci U S A 100:7348-7353. CrossRef Medline

Niwa M, Johnson JS, O'Connor KN, Sutter ML (2012a) Activity related to perceptual judgment and action in primary auditory cortex. J Neurosci 32:3193-3210. CrossRef Medline

Niwa M, Johnson JS, O'Connor KN, Sutter ML (2012b) Active engagement improves primary auditory cortical neurons' ability to discriminate temporal modulation. J Neurosci 32:9323-9334. CrossRef Medline

Niwa M, Johnson JS, O'Connor KN, Sutter ML (2013) Differences between primary auditory cortex and auditory belt related to encoding and choice for AM sounds. J Neurosci 33:8378-8395. CrossRef Medline

O'Connor KN, Barruel P, Sutter ML (2000) Global processing of spectrally complex sounds in macaques (Macaca mullata) and humans. J Comp Physiol A Neuroethol Sens Neural Behav Physiol 186:903-912. CrossRef Medline
O’Connor KN, Yin P, Petkov CI, Sutter ML (2010) Complex spectral interactions encoded by auditory cortical neurons: relationship between bandwidth and pattern. Front Syst Neurosci 4: 145:1-16. CrossRef Medline

O'Connor KN, Johnson JS, Niwa M, Noriega NC, Marshall EA, Sutter ML (2011) Amplitude modulation detection as a function of modulation frequency and stimulus duration: comparisons between macaques and humans. Hearing Res 277:37-43. CrossRef Medline

Oram MW, Földiák P, Perrett DI, Sengpiel F (1998) The "Ideal Homunculus": decoding neural population signals. Trends Neurosci 21:259-265. CrossRef Medline

Romo R, Hernández A, Zainos A, Salinas E (2003) Correlated neuronal discharges that increase coding efficiency during perceptual discrimination. Neuron 38:649-657. CrossRef Medline

Rothschild G, Nelken I, Mizrahi A (2010) Functional organization and population dynamics in the mouse primary auditory cortex. Nat Neurosci 13:353-360. CrossRef Medline

Scheich H, Brechmann A, Brosch M, Budinger E, Ohl FW (2007) The cognitive auditory cortex: task-specificity of stimulus representations. Hearing Res 229:213-224. CrossRef Medline

Shadlen MN, Newsome WT (1998) The variable discharge of cortical neurons: implications for connectivity, computation, and information coding. J Neurosci 18:3870-3896. Medline

Smith MA, Kohn A (2008) Spatial and temporal scales of neuronal correlation in primary visual cortex. J Neurosci 28:12591-12603. CrossRef Medline

Wang X, Lu T, Snider RK, Liang L (2005) Sustained firing in auditory cortex evoked by preferred stimuli. Nature 435:341-346. CrossRef Medline

Winkowski DE, Bandyopadhyay S, Shamma SA, Kanold PO (2013) Frontal cortex activation causes rapid plasticity of auditory cortical processing. J Neurosci 33:18134-18148. CrossRef Medline

Yin P, Johnson JS, O'Connor KN, Sutter ML (2011) Coding of amplitude modulation in primary auditory cortex. J Neurophysiol 105:582-600. CrossRef Medline

Zohary E, Shadlen MN, Newsome WT (1994) Correlated neuronal discharge rate and its implications for psychophysical performance. Nature 370:140-143. CrossRef Medline 